\title{
Some remarks on algebraic rings
}

To Professor Y. Akizuki on his 60-th birthday

By

Hiroshi Yanagihara

(Communicated by Prof. Nagata, Aug. 5, 1963)

1. In the paper [1], Greenberg called a unitary ring $R$ to be an algebraic ring defined over $k$ if the following conditions are satisfied :

1) $R$ is a union of a finite number of algebraic varieties defined over $k$.

2) $R$ is an algebraic group defined over $k$ as to its additive law.

3) The mapping of $R \times R$ onto $R$, which maps $(a, b)$ onto $a b$, is an everywhere regular mapping defined over $k$.

4) The unit 1 of $R$ is a rational point of $R$ over $k$.

5) The set $U$ of the units in $R$ is a locally k-closed subset in $R$.

6) The mapping of $U$ onto $U$, which maps a onto $a^{-1}$, is an everywhere regular mapping on $U$.

In this note we shall remark, first, that if $R$ is an algebraic ring defined over $k$ in the above sense, then the set $U$ of the units in $R$ is a k-open subset of $R$, and that if the characteristic of $k$ is zero, the conditions 5) and 6) can be excluded from the definition of an algebracic ring, i.e., if $R$ satisfies 1 ), 2), 3) and 4), then $R$ satisfies necessarily 5) and 6). Let $R$ be an algebraic ring defined over $k$. Then a two-sided ideal $I$ of $R$ will be called an algebric ideal of $R$ if $I$ is a closed subset of $R$. Then we shall construct a residue class ring of $R$ by an algebraic ideal, which is also an algebraic ring. Lastly we shall show that if $R$ is connected, any two-sided ideal of $R$ is a connected algebraic ideal and $R$ is a ring with maximal and minimal conditions for two-sided ideals.

2. Let $R$ be a unitary ring which satisfies the conditions 1 ), 2), 
3) and 4), and $U$ the set of the units in $R$. Let $R_{0}, R_{1}, \cdots, R_{s-1}$ be the components of $R$, where 1 is contained in $R_{1}$. When $a$ is any element of $U$, let $R_{i}$ and $R_{j}$ be the components which contain $a$ and $a^{-1}$ respectively, and let $\Gamma_{i j}$ be the graph of the mapping $f_{i j}$ of $R_{i} \times R_{j}$ into $R_{1}$ which maps $(a, b)$ onto $a b$.

Lemma 1. Let $R, U, a, \Gamma_{i j}$ be as above. Then we have the following :

(i) $k\left(a, a^{-1}\right)$ is purely inseparable over $k(a)$.

(ii) $\mathrm{I}_{i j} \cap\left(R_{i} \times R_{j} \times 1\right)$ has only one component, which is the locus of $\left(x, x^{-1}, 1\right)$ over $\bar{k}$, where $x$ is any generic point of $R_{i}$ over $k$.

(iii) $R_{i} \cap U$ is a dense subset of $R_{i}$.

Proof. If ( $\mathrm{i}$ ) is not true, there is a point $b$ different from $a^{-1}$ such that $b$ is a generic specialization of $a^{-1}$ over $k(a)$. Then $a b=f_{i j}(a, b)=f_{i j}\left(a, a^{-1}\right)=a a^{-1}=1$, since $f_{i j}$ is defined over $k$. This is a contradiction. Therefore (i) is true. Since $\left(a, a^{-1}, 1\right)$ is a point of $\Gamma_{i j} \cap\left(R_{i} \times R_{j} \times 1\right)$, this set is not empty. Let $n$ be the dimension of $R$. Then it is easy to see that the dimension of any component $C$ of $\mathrm{L}_{i j} \cap\left(R_{i} \times R_{j} \times 1\right)$ is not less than $n$, since $R_{i} \times R_{j} \times R_{1}$ is non-singular. If $(x, y, 1)$ is a generic point of $C$ over $\bar{k}, y$ is equal to $x^{-1}$. Therefore $k(x, y)$ is purely inseparable over $k(x)$ by (i), and $x$ is a generic point of $R_{i}$ over $k$. This means that $\Gamma_{i j} \cap$ $\left(R_{i} \times R_{j} \times 1\right)$ has only one component and that $R_{i} \cap U$ contains any generic point of $R_{i}$ over $k$. Therefore (ii) and (iii) are also satisfied.

q.e.d.

PROPOSITION 1. Let $R$ be an algebraic ring defined over $k$. Then the set $U$ of the units of $R$ is a k-open subset of $R$.

Proof. Let $R_{i}$ be a component of $R$ which intersects with $U$. Then by Lemma $1 R_{i} \cap U$ is a dense subset of $R_{i}$. On the other hand $U$ is a locally closed subset of $R$, and hence $R_{i} \cap U$ must be a $k$-open subset of $R_{i}$. Therefore $U$ is a $k$-open subset of $R$, since $R$ is a disjoint sum of $R_{0}, R_{1}, \cdots, R_{s-1}$.

q.e.d.

PROPOSITION 2. Let $R$ be a unitary ring which satisfies the conditions 1$), 2), 3$ ) and 4). Let $R_{1}$ be the component containing 1 , and $x$ a generic point of $R_{1}$ over $k$. Then if $k\left(x^{-1}\right)$ is equal to $k(x)$, 
$R$ is an algebraic ring defined over $k$, i.e., $R$ satisfies the conditions 5) and 6).

ProOF. Let $R_{0}, R_{1}, \cdots, R_{s-1}$ be the components of $R$. Let $f_{i j}$ and $\Gamma_{i j}$ be the same as in Lemma 1. By the assumptions, the locus $V$ of $\left(x, x^{-1}\right)$ over $k$ on $R_{1} \times R_{1}$ defines a birational correspondence $g$ between $R_{1}$ and itself. If $a$ is any point of $R_{1} \cap U, a^{-1}$ is also in $R_{1} \cap U$. Then $\left(a, a^{-1}, 1\right)$ is in $\Gamma_{11} \cap\left(R_{1} \times R_{1} \times 1\right)$ and hence $\left(a, a^{-1}\right)$ is in $V$ by (ii) of Lemma 1 . Moreover it is easy to see that if $(a, b)$ is in $V, b$ is equal to $a^{-1}$. Therefore $g$ is regular at $a$ by Zariski's Main Theorem, since $R_{1}$ is nonsingular. Similarly $g^{-1}$ is regular at $a^{-1}$. On the other hand if $a$ is any point of $R_{1}$ such that $(a, b)$ is in $V$ for some $b$ in $R_{1}, a$ is in $R_{1} \cap U$ by (ii) of Lemma 1. Therefore $R_{1} \cap U$ is the set of the points at which $g$ is biregular and hence it is a $k$-open subset of $R_{1}$. Next let $R_{i}$ be a component of $R$ which intersects with $U$. Then any generic point $x_{i}$ of $R_{i}$ is in $U$ by (iii) of Lemma 1 . Assume that $x_{i}^{-1}$ is in $R_{j}$, and let $x_{j}$ be a generic point of $R_{j}$ over $k\left(x_{i}\right)$. Then $x_{i} x_{j}$ is in $R_{1}$ and hence $R_{1} \cap U$. Therefore we have $k\left(x_{i}, x_{j}\right)>k\left(x_{i} x_{j}\right)=k\left(\left(x_{i} x_{j}\right)^{-1}\right)$. On the other hand, since $x_{j}\left(x_{i} x_{j}\right)^{-1}=x_{i}^{-1}$, we have $k\left(x_{i}, x_{j}\right)>k\left(x_{i}^{-1}, x_{j}\right)$ and hence $k\left(x_{i}\right)>k\left(x_{i}^{-1}\right)$. Similarly we have $k\left(x_{i}^{-1}\right)>k\left(x_{i}\right)$. Therefore $k\left(x_{i}\right)$ is equal to $k\left(x_{i}^{-1}\right)$, and we can define a birational correspondence $g_{i j}$ between $R_{i}$ and $R_{j}$. By the quite similar way to the above, we can see that $R_{i} \cap U$ is a $k$-open subset of $R_{i}$ and $g_{i j}$ is biregular at any point of $R_{i} \cap U$. This means that the conditions 5) and 6) are slso satisfied on $R$.

q.e.d.

COROLlary. Let $R$ be a unitary ring which satisfies the conditions 1), 2), 3) and 4). Then if the characteristic of $k$ is zero, $R$ is an algebraic ring defined over $k$.

Proof. This is a direct consequence of Proposition 2 and Lemma 1.

3. Now we shall call an algebraic ring $S$ to be a residue class ring of an algebraic ring $R$ by an algebraic ideal $I$ of $R$ (see 1) if $S$ satisfies the following condition: 
There is a separable homomorphism $\rho$ of $R$ onto $S$, whose kernel is equal to $I$.

Remark: $S$ is a factor group of $R$ by $I$ with respect to additive law in the sense of [2]. It is easy to see that the residue class ring of $R$ by $I$ is uniquely determined within a biregular isomorphism.

THEOREM 1. Let $R$ be an algebraic ring defined over $k$ and $I$ an algebraic ideal of $R$ whose components are defined over $k$. Then there exists always a residue class ring of $R$ by $I$, which is defined over $k$.

Proof. By Theorem 4 in [2], there exists a factor group $S=R / I$ of $R$ by $I$, defined over $k$, with respect to additive law. Let $\rho$ be the natural homomorphism of $R$ onto $S$ with the kernel $I$. We shall show that $S$ has a structure of an algebraic ring defined over $k$, which satisfies the condition of a residue class ring of $R$ by $I$. Let $S_{1}$ and $S_{2}$ be two components of $S\left(S_{1}\right.$ may be equal to $S_{2}$ ), and let $R_{1}$ and $R_{2}$ be components of $R$ which are mapped onto $S_{1}$ and $S_{2}$ by $\mathcal{P}$ respectively. If $x$ and $y$ are independent generic points of $R_{1}$ and $R_{2}$ over $k$ respectively, $\phi(x)$ and $\varphi(y)$ are independent generic points of $S_{1}$ and $S_{2}$ over $k$ respectively. Now we show that $k(\mathcal{P}(x), \mathcal{P}(y))$ contains $k(\mathcal{P}(x y))$. In fact $k(\varphi(x y)$, $\varphi(x), \varphi(y))$ is separable over $k(\varphi(x), \varphi(y))$, since $k(x, y)$ contains $k(\mathcal{P}(x y))$ and $k(x, y)$ is separable over $k(\mathcal{P}(x), \varphi(y))$. If $k(\varphi(x), \varphi(y))$ does not contain $k(\varphi(x y))$, there exists a generic specialization $z$, different from $\mathcal{P}(x y)$, of $\mathcal{P}(x y)$ over $k(\mathcal{P}(x), \mathcal{P}(y))$, which can be extended to a generic specialization $\left(x^{\prime}, y^{\prime}\right)$ of $(x, y)$. Then we have $z=\mathcal{P}\left(x^{\prime} y^{\prime}\right), \mathcal{P}\left(x^{\prime}\right)=\mathcal{P}(x)$ and $\mathcal{P}\left(y^{\prime}\right)=\mathscr{P}(y)$. From these, we conclude that $x-x^{\prime}$ and $y-y^{\prime}$ are both in $I$ and hence that $x y-x^{\prime} y^{\prime}$ is in $I$. This means that $z=\mathscr{P}\left(x^{\prime} y^{\prime}\right)=\mathscr{P}(x y)$, and we have a contradiction. Therefore we have a rational mapping $h^{\prime}$ of $S_{1} \times S_{2}$ into a component $S_{3}$ of $S$ which maps $(\mathcal{P}(x), \mathcal{P}(y))$ onto $\varphi(x y)$. Assume that $x y$ is in a component $R_{3}$ of $R$, and let $h$ be the rational mapping of $R_{1} \times R_{2}$ into $R_{3}$ which maps $(x, y)$ onto $x y$. Let $\left(a^{\prime}, b^{\prime}\right)$ be any point of $S_{1} \times S_{2}$. Then the specialization $(\mathscr{P}(x), \varphi(y)) \stackrel{k}{\rightarrow}\left(a^{\prime}, b^{\prime}\right)$ can always be extended to a specialization $(x, y) \stackrel{k}{\rightarrow}(a, b)$. Then $(x y, \mathcal{P}(x y))$ has 
a uniquely determined specialization $(a b, \mathcal{P}(a b))$ over the above specialization. Moreover we can easily see that $\mathcal{P}(a b)$ depends only on $\left(a^{\prime}, b^{\prime}\right)$, but not on $(a, b)$. From this fact we can see, using Zariski's Main Theorem, that $h^{\prime}$. is regular at $\left(a^{\prime}, b^{\prime}\right)$. Therefore we easily see that $S$ has a structure of a ring whose multiplication is defined naturally by that of $R$. Then $\mathcal{P}$ is a ring homomorphism of $R$ onto $S$, and $S$ satisfies the conditions 1 ), 2),3) and 4). Now let $R_{1}$ (resp. $S_{1}$ ) be the component of $R$ (resp. $S$ ) containing 1 . Then $\varphi\left(R_{1}\right)$ is equal to $S_{1}$. Let $x$ be a generic point of $R_{1}$. Then we have $k(x)=k\left(x^{-1}\right)>k\left(\mathcal{P}\left(x^{-1}\right)\right)=k\left(\mathcal{P}(x)^{-1}\right)$. On the other hand $k(x)$ is separable over $k(\mathcal{P}(x))$ and $k\left(\mathcal{P}(x), \varphi(x)^{-1}\right)$ is purely inseparable over $k(\mathscr{P}(x))$ by Lemma 1 . Therefore we have $k(\mathcal{P}(x))=k\left(\mathcal{P}(x)^{-1}\right)$ and hence $S$ is an algebraic ring defined over $k$ by Proposition 2 .

q.e.d.

Remark: Let $R$ be an algebraic ring and $I$ its algebraic ideal. If the residue class ring $R / I$ is connected, the set $U$ of the units in $R$ is mapped onto the set $U^{\prime}$ of the units in $R / I$. In fact let $R_{1}$ be the component of $R$ containing 1 . Then $\left.\varphi\right|_{U \cap R_{1}}$ is a rational homomorphism of $U \cap R_{1}$ into $U^{\prime}$, which is defined over $k$ and generically surjective. This means that $\mathcal{P}$ maps $U \cap R_{1}$ onto $U^{\prime}$.

4. In the following we shall study the structure of algebraic ideals of a connected algebraic ring.

PROPOSITION 3. Let $R$ be an algebraic ring defined over $k$ and $I$ an algebraic ideal of $R$. Then the component $I_{0}$ of I containing 0 is also an algebraic ideal of $R$. In particular the component $R_{0}$ of $R$ containing 0 is an algebraic ideal of $R$.

Proof. Let $R_{i}$ be any component of $R$ and $k^{\prime}$ a field containing $k$ over which all the components of $I$ are defined. Let $x_{i}$ and $y$ be independent generic points of $R_{i}$ and $I_{0}$ over $k^{\prime}$. It is sufficient to show that $x_{i} y$ and $y x_{i}$ are both in $I_{0}$. Since $I$ is an algebraic ideal of $R, x_{i} y$ and $y x_{i}$ are in components of $I$. On the other hand $y$ is specialized to 0 over $k^{\prime}$ and hence $x_{i} y$ (resp. $y x_{i}$ ) is specialized to 0 over $k^{\prime}$. Therefore $x_{i} y$ are $y x_{i}$ must be in $I_{0}$. 
COROLlary. Let $R$ be an algebraic ring defined over $k$. Then $R$ is connected if and only if the components containing 0 and 1 are the same.

PROPOSITION 4. Let $R$ be a connected algebraic ring defined over $k$. Then any algebraic ideal of $R$ is also connected.

Proof. Let $I$ be an algebraic ideal of $R$, and let $k^{\prime}$ be a field containing $k$ over which all the components of $I$ are defined. Let $I_{i}$ be any component of $I$. If $x$ and $y_{i}$ are independent generic points of $R$ and $I_{i}, x y_{i}$ is in a component $I_{j}$ of $I$. However $x$ has 1 as a specialization over $k^{\prime}, x y_{i}$ is in $I_{i}$, i.e., $I_{j}$ is equal to $I_{i}$. On the other hand $x$ has 0 as a specialization over $k^{\prime}, x y_{i}$ is in the component $I_{0}$ of $I$ containing 0 , i.e., $I_{j}$ is equal to $I_{0}$. This means that $I_{i}$ is equal to $I_{0}$.

COROLlary. Let $R$ be a connected algebraic ring. Then the length $m$ of a sequence of proper algebraic ideals of $R$ such that

$$
I_{1} \subsetneq I_{2} \subsetneq \cdots \subsetneq I_{m}
$$

is less than the dimension of $R$.

PROPOSITION 5. Let $R$ be a connected algebraic ring defined over $k$. Let $a_{1}, a_{2}, \cdots, a_{s}$ be rational points of $R$ over $k$. Then the twosided ideal generated by $a_{1}, a_{2}, \cdots, a_{s}$ is a connected algebraic ideal of $R$, whose component is defined over $k$.

Proof. Let $x_{1}, \cdots, x_{s}, y_{1}, \cdots, y_{s}$ be independent generic points of $R$ over $k$, and put $z=x_{1} a_{1} y_{1}+\cdots+x_{s} a_{s} y_{s}$. Then $k(z)$ is a regular extension over $k$. Let $W$ be the locus of $z$ over $k$. Let $z_{1}, \cdots, z_{i}$ be independent generic points of $W$ over $k$ and $W_{i}$ the locus of $z_{1}+\cdots+z_{i}$ over $k$. Since $W$ contains 0 , we have $W_{i} \subset W_{i 1_{1}}$ and hence there exists a positive integer $N$ such that $W_{N}=W_{i}$ for any $i \geqq N$. Then it is easy to see that $W_{N}$ is an algebraic subgroup of $R$ as an additive group (cf. Propositions 3 and 5 in [3]) and that $W_{N}$ contains the ideal $I$ generated by $a_{1}, \cdots, a_{s}$ as a dense subset. Since $W_{N}$ is an algebraic subgroup of $R$, any point in $W_{N}$ is the sum of two generic points of $W_{N}$ over $k$ and hence is a 
point of the ideal $I$. Therefore $W_{N}$ is equal to $I$, i.e., $I$ is an algebraic ideal.

q.e.d.

We shall say that an algebraic ideal $I$ of $R$ is defined over $k$, if $k$ is a field of definition of $R$ and all the components of $I$.

COROLlary. Let $R$ be a commutative algebraic ring defined over an algebraically closed field $k$. If $R$ is not a field, then $R$ has a proper algebraic ideal defined over $k$.

Proof. If $R$ is not connected, the component of $R$ containing 0 is a proper algebraic ideal defined over $k$ by Proposition 3. If $R$ is connected, there is a rational point $a$ over $k$ such that $a$ is not a unit of $R$. Then $a R$ is a proper algebraic ideal defined over $k$ by Proposition 5 .

q.e.d.

THEOREM 2. Let $R$ be a connected algebraic ring defined over an algebraically closed field $k$. Then for any algebraic ideal $I$ of $R$ defined over $k$, there are rational points $a_{1}, \cdots, a_{s}$ such that $I=$ $\left(a_{1}, \cdots, a_{s}\right)$. Moreover the number $s$ of the points can be less than the dimension of $R$.

Ppoof. Let $a_{1}, \cdots, a_{i}$ be any rational points of $I$ over $k$. Then $\left(a_{1}, \cdots, a_{i}\right)$ is in $I$. Therefore we can choose a sequence of rational points $a_{1}, a_{2}, \cdots, a_{i}, \cdots$, of $I$, such that if $\left(a_{1}, \cdots, a_{i-1}\right)$ is not equal to $I, a_{i}$ is not in $\left(a_{1}, \cdots, a_{i-1}\right)$. This is possible, since the set of the rational points in $I$ over $k$ is dense in $I$. This means that our assetion is true by Corollary of Proposition $4 . \quad$ q.e.d.

THEOREM 3. Let $R$ be a connected algebraic ring defined over $k$. Then any ideal of $R$ is an algebraic ideal defined over a field containing $k$ and $R$ is a ring with maximal and minimal conditions for two-sided ideals.

Proof. First notice that, for any finite set of points $a_{1}, \cdots, a_{t}$ in $R,\left(a_{1}, \cdots, a_{t}\right)$ is an algebraic ideal of $R$ defined over $k\left(a_{1}, \cdots, a_{t}\right)$ by Corollary of Proposition 5. From this we can see that $R$ is a ring with maximal condition for two-sided ideals. In fact if it is not so, there exists an ideal $I$ of $R$ which is not finitely generated. Then we can choose $a_{i}(i=1,2, \cdots)$ inductively so that $a_{i}$ is an 
element of $I$ not contained in $I_{i-1}=\left(a_{1}, \cdots, a_{i-1}\right)$. On the other hand $I_{j}$ is a subvariety of $R$ defined over $k\left(a_{1}, \cdots, a_{i}\right)$ for $j \leqq i$. Therefore by Corallary of Proposition 4 there exists a positive integer $N$ such that $I_{i}=I_{N}$ for $i \geqq N$. This is a contradiction. Since any two-sided ideal $I$ of $R$ is finitely generated, $I$ is an algebraic ideal of $R$ defined over a certain field containing $k$ by Corollary of Proposition 5 and hence the length of any sequence of proper twosided ideals such that

$$
I_{1} \supsetneq I_{2} \supsetneq \cdots \supsetneq I_{m}
$$

is less than the dimension of $R$ by Corollary of Proposition 4. q.e.d.

Remark: Although we treat only two-sided ideals of connected algebraic rings in Theorems 2 and 3, similar results can be obtained in the case of left (or right) ideals. In other words any left (or right) ideals of a connected algebraic ring $R$ is closed in a Zariski topology and $R$ is a ring with maximal and minimal conditions for left (or right) ideals. In fact we can easily see that all results in this section are modified, taking closed left (or right) ideals instead of algebraic ideals.

\section{FACUlTy OF SCIENCE, HiRoshima University.}

\section{REFERENCES}

[1] Greenberg, M. J., "Schemata over local rings", Ann. of Math., Vol. 73 (1961), pp. 624-648.

[2] Rosenlicht, M., "Some basic theorems on algebraic groups", Amer. J. of Math., Vol. 78 (1956), pp. 401-443.

[3] Weil, A., "Variétés abéliennes et courbes algébriques", Actualités sci. et ind. no. 1064 (1948), Paris. 